\title{
Efficient Approach to Superior Vena Cava Baffle Stenosis Following the Mustard Procedure: Expanding Role of a Mechanical Rotating Dilator Sheath for Lead Extraction
}

\author{
Chi Chi Do-Nguyen ${ }^{1}$, Alexander Ochman², Maxwell Kilcoyne ${ }^{1}$, Richard Kovach ${ }^{2}$, Boban \\ Abraham $^{3}$, Pedram Kazemian ${ }^{2}$, Lynn McGrath ${ }^{2}$, and Randy Stevens ${ }^{3}$ \\ ${ }^{1}$ Philadelphia College of Osteopathic Medicine \\ ${ }^{2}$ Deborah Heart and Lung Center \\ ${ }^{3}$ Saint Christopher's Hospital for Children
}

April 28, 2020

\begin{abstract}
Introduction: In adult congenital patients with transposition of the great arteries originally treated with the Mustard (atrial switch) procedure, the most common reason for re-intervention is baffle stenosis. This may be exacerbated by permanent transvenous pacemaker lead placement across the baffle. Case Report: A 47-year-old female status post Mustard procedure performed at 15 months old presented with a high-grade stenosis of the superior vena cava (SVC) baffle from the SVC to the left atrium, with a nonfunctional permanent pacemaker lead passing through the baffle. A mechanical rotating dilator sheath was used for attempted lead extraction, relieving the baffle stenosis almost completely as a secondary effect, prior to the placement of a $10 \times 27 \mathrm{~mm}$ Visipro balloon expandable stent in the SVC baffle. Conclusions: Use of the mechanical rotating dilator sheath is an evolving treatment strategy in adult congenital heart disease to minimize the risk of bleeding, trauma to surrounding structures, and death. Its ability to fully alleviate baffle stenosis even when full lead extraction is not feasible or is associated with significant procedural risk further demonstrates its expanded role in this patient population. A multidisciplinary approach and great diligence must be employed to avoid potential complications.
\end{abstract}

\section{INTRODUCTION}

In adult congenital patients with transposition of the great arteries (TGA) originally treated with the Mustard (atrial switch) procedure, long term complications include baffle stenosis, arrhythmias, and sudden death. ${ }^{1}$ The re-intervention rate for these patients is as high as $9.7 \%$, with the most common reason being baffle stenosis. ${ }^{2}$ Permanent pacemakers are required in this patient population for management of symptomatic sinus node or AV node dysfunction and the presence of transvenous leads across the baffle can increase stenosis rates up to $58 \% \%^{1,3}$ This report describes a case of multidisciplinary lead extraction and recanalization of an occluded superior vena cava (SVC) baffle using a mechanical rotating dilator sheath in a patient with a prior Mustard procedure and retained left atrium (LA) pacemaker lead.

\section{CASE REPORT}

A 47-year-old female with a past medical history notable for complete d-TGA and an extensive interventional history (Table 1 ) presented with SVC syndrome. An echocardiogram revealed a high-grade stenosis of the SVC baffle from the SVC to the LA, with a mean gradient of $5 \mathrm{mmHg}$ and remaining luminal area of approximately $1 \mathrm{~mm}$. A nonfunctional permanent pacemaker lead passing through the baffle and located in the LA was contributing to the obstruction and the patient was scheduled for extraction of the LA lead and balloon angioplasty and stenting of the SVC baffle. 


\section{Surgical Planning}

In a hybrid operating room, 6 and 7 French (Fr) sheaths were advanced percutaneously using a micropuncture technique into the right common femoral artery and vein, respectively. A 6 Fr sheath and a 10 Fr multi lumen venous sheath was advanced similarly into the left common femoral artery and vein, respectively. In the event emergent bypass was needed, a 4 Fr sheath was advanced into the left superficial femoral artery and a 9 Fr sheath was advanced under ultrasound guidance into the right internal jugular (RIJ) vein. The patient was then partially anticoagulated.

Cardiac catheterization demonstrated no flow through the SVC baffle, with venous drainage from the upper body traveling through a massively dilated hemi-azygous vein in communication with the inferior vena cava (IVC), which drains into the LA via the IVC baffle. The IVC baffle had no signs of obstructive disease (Figure 1 ).

\section{Multidisciplinary Lead Extraction and Baffle Obstruction Relief}

Manual traction of the non-functioning LA lead demonstrated complete attachment to the subclavian vein and SVC. To increase the safety of the extraction procedure and to provide hemostasis in case of an SVC tear, an occlusive balloon (Bridge Occlusion Balloon 590-001, Philips) was deployed in the SVC by way of the right internal jugular vein. Attempt retracting the active fixation mechanism of the lead was unsuccessful. A locking stylet and short-length mechanical dilating rotator sheath (9F TightRail Sub-C 560-009, Philips) was used to lyse the adhesions from the entrance of the lead into the subclavian vein. The dilator was exchanged for a long rotational sheath (9F TightRail 545-509, Philips) (Figure 2 ) and the adhesions for the rest of the lead from the turn of the SVC into the baffle were released. The tip of the lead was firmly embedded inside the atrial baffle and could not be safely extracted despite multiple attempts (Figure 3A ). The use of the mechanical dilating rotator sheath almost completely relieved the stenosis at the baffle ostium as a side effect (Figure 3B ). Due to resolution of stenosis, lack of progress, and an increasing risk of perforation, a decision was made not to proceed with complete extraction of the lead. The lead was cut and capped near the entry into the subclavian vein and the procedural site was closed. The patient was then fully anticoagulated.

A $10 \times 27 \mathrm{~mm}$ Visipro balloon expandable stent was advanced into the RIJ vein, positioned safely across the stenotic segment of the baffle, and deployed with a 14-mm balloon (Figure 3C ). Repeat angiography demonstrated very brisk flow from the SVC through the baffle with no residual pressure gradient (Figure 3D ). Anticoagulation was reversed and the stent delivery system was removed.

At her one month follow up, the patient remained on dofetilide and Eliquis for rate control and anticoagulation, respectively, with no symptoms nor complications.

\section{DISCUSSION}

Stenosis of the SVC baffle occurs in up to $40 \%$ of patients treated with the Mustard procedure, with risk factors including a Dacron baffle, a tortuous baffle course, baffle leaks, and a young age at operation..$^{1,2,4,5}$ With the increase in permanent pacemaker insertion in this patient population, transvenous lead placements across the baffle may increase stenosis rates to up to $58 \% .^{1,3}$ Baffle stenosis can lead to severe complications, such as persistent hemodynamic load, hypoxia, thromboembolic events, and SVC syndrome, requiring reintervention. $^{3}$

Transcatheter interventions have been the preferred therapeutic option in transvenous lead extraction and relief of baffle obstructions, including laser lead extraction or radiofrequency perforation followed by angioplasty and stent placement. ${ }^{2-4,6,7}$ Laser lead extraction uses pulsatile ultraviolet light to dissolve fibrous tissue, whereas radiofrequency perforation uses rapid heating. ${ }^{6-8}$ However, both methods are associated with risks of cardiac wall perforation and damage to surrounding structures or the conduction system. ${ }^{6,7}$

In adult congenital heart disease (CHD), use of the mechanical rotating dilator sheath is an evolving treatment strategy to minimize the risk of bleeding, trauma to surrounding structures, and death. The baffle 
stenosis is typically more compliant than native tissue stenosis. Therefore, great diligence is required with the mechanical rotating dilator sheath to avoid over-dilation and puncture through the baffle wall. ${ }^{2}$ Intraprocedural angiography aids in guiding dilatation and maintaining safety. Complete transvenous lead extraction in CHD patients has been successful in up to $92 \%$ of patients in prior studies, with failures attributed to calcified adhesions or active fixation..$^{3,6,9,10}$ In our case, we recognized the risks associated with completely removing the nonfunctional LA lead. Therefore, extraction was halted.

A second benefit of using the mechanical rotating dilator sheath was its ability to fully alleviate the SVC baffle stenosis. A stent may not have been necessary after attempted lead extraction in this case due to the low gradient shown after adhesiolysis alone. However, recognizing that delaying baffle stenting may lead to rapid thrombosis, especially after extensive adhesiolysis, we continued with deployment of the balloonexpandable stent. ${ }^{3}$ Optimal stent placement is crucial for future pacemaker lead placement, which may be necessary in the case of this patient, as well as the benefits of decreased baffle gradients, increased baffle diameter, relief of clinical symptoms, and delay of the need for re-intervention. ${ }^{2}$

A multi-disciplinary team approach including an interventional cardiology, electrophysiology, and cardiothoracic surgical team that are familiar with adult CHD is crucial in managing these complex cardiac cases safely. Prophylactic measures are taken to avoid complications, such as intubation, full sedation, and placement of a transesophageal echocardiogram (TEE) probe. During the procedure, obtaining access from the bilateral femoral arteries and veins allows for accurate localization of the baffle from above and below, as well as continuous imaging during stent placement. ${ }^{2}$ Further, obtaining access for emergent bypass and placing a sheath and balloon in the right internal jugular vein for emergent tamponade are necessary in procedural planning, as the periprocedural mortality rate for baffle-related re-intervention can be as high as $29 \% .{ }^{2,5,11}$ Additionally, the extent and timing of anticoagulation is pre-planned to avoid excessive bleeding throughout the course of the combined procedure.

\section{CONCLUSION}

In patients with TGA previously treated with the Mustard procedure, the high prevalence of long term complications due to transvenous pacing and baffle stenosis leads to clinically challenging situations. This report represents the outcomes of a unique, safe, and feasible strategy used in dilation of baffle stenosis using a mechanical rotating dilator sheath, followed by balloon-expandable stenting of the SVC baffle with a jailed non-functioning LA lead. Further studies are needed to assess the efficacy and safety of the mechanical rotating dilator sheath in other settings of CHD.

\section{REFERENCES}

1. Bottega NA, Silversides CK, Oechslin EN, et al. Stenosis of the superior limb of the systemic venous baffle following a Mustard procedure: An under-recognized problem. International Journal of Cardiology. 2012; 154(1): 32-37.

2. Hill KD, Fleming G, Curt Fudge J, Albers EL, Doyle TP, Rhodes, JF. Percutaneous interventions in high-risk patients following mustard repair of transposition of the great arteries. Cathet Cardiovasc Intervent. 2012; 80: 905-914.

3. Laredo M, Waldmann V, Chaix MA, et al. Lead Extraction With Baffle Stenting in Adults With Transposition of the Great Arteries. JACC Clin Electrophysiol. 2019; 5(6): 671-680.

4. Love B, Mehta D, Fuster V. Evaluation and management of the adult patient with transposition of the great arteries following atrial-level (Senning or Mustard) repair. Nat Rev Cardiol. 2008; 5: 454-467.

5. MacLellan-Tobert SG, Cetta F, Hagler DJ. Use of Intravascular Stents for Superior Vena Caval Obstruction After the Mustard Operation. Mayo Clinic Proceedings. 1996; 71(11): 1071-1076.

6. Khairy P, Roux JF, Dubuc M, et al. Laser Lead Extraction in Adult Congenital Heart Disease. Journal of Cardiovascular Electrophysiology. 2007; 18: 507-511.

7. Asgar AW, Mirò J, Ibrahim R. Recanalization of systemic venous baffles by radiofrequency perforation and stent implantation. Cathet. Cardiovasc. Intervent. 2007; 70: 591-594.

8. Simpson L, Bhella PS, Schussler JM, Grayburn PA, Assar M. Pacemaker laser lead extraction and 
reimplantation of dual-chamber implantable cardioverter defibrillator via Mustard baffle in complete transposition of great arteries. Proc (Bayl Univ Med Cent). 2010; 23(3): 256-258.

9. McCanta AC, Kong MH, Carboni MP, Greenfield RA, Hranitzky PM, Kanter RJ. Laser Lead Extraction in Congenital Heart Disease: A Case-Controlled Study. Pacing and Clinical Electrophysiology. 2013; 00: 1-9.

10. Crossley GH, Sorrentino RA, Exner DV, et al. Extraction of chronically implanted coronary sinus leads active fixation vs passive fixation leads. Heart Rhythm. 2016; 13(6): 1253-1259.

11. Bradley EA, Cai A, Cheatham SL, et al. Mustard baffle obstruction and leak - How successful are percutaneous interventions in adults? Progress in Pediatric Cardiology. 2015; 39: 157-163.

12. TightRail rotating mechanical dilator sheath product brochure. San Diego, CA; Phillips; Published 2018. https://philipsproductcontent.blob.core.windows.net/assets/20180718/568a170a8e5a4dc2922fa921014449ed.pdf. Accessed April 13, 2020.

\begin{tabular}{|l|l|l|}
\hline Year & Age & Procedure \\
\hline 1972 & $0-1$ month & Balloon atrial septostomy \\
\hline 1973 & 15 months & Mustard (atrial switch) procedure \\
\cline { 3 - 3 } & Reoperation to relieve inferior vena cava obstruction \\
\hline 2006 & 34 years & $\begin{array}{l}\text { Placement of atrial demand pacing (AAIR) pacemaker secondary to } \\
\text { sinoatrial node dysfunction }\end{array}$ \\
\hline 2018 & 46 years & $\begin{array}{l}\text { Pacemaker extraction with capping of LA lead. Patient was noted to be in } \\
\text { elective replacement indicator (ERI) mode and was not pacer dependent }\end{array}$ \\
\hline 2019 & 47 years & $\begin{array}{l}\text { Initiated on dofetilide and successful cardioversion due to atrial fibrillation, } \\
\text { resulting in return to sinus rhythm }\end{array}$ \\
\hline
\end{tabular}



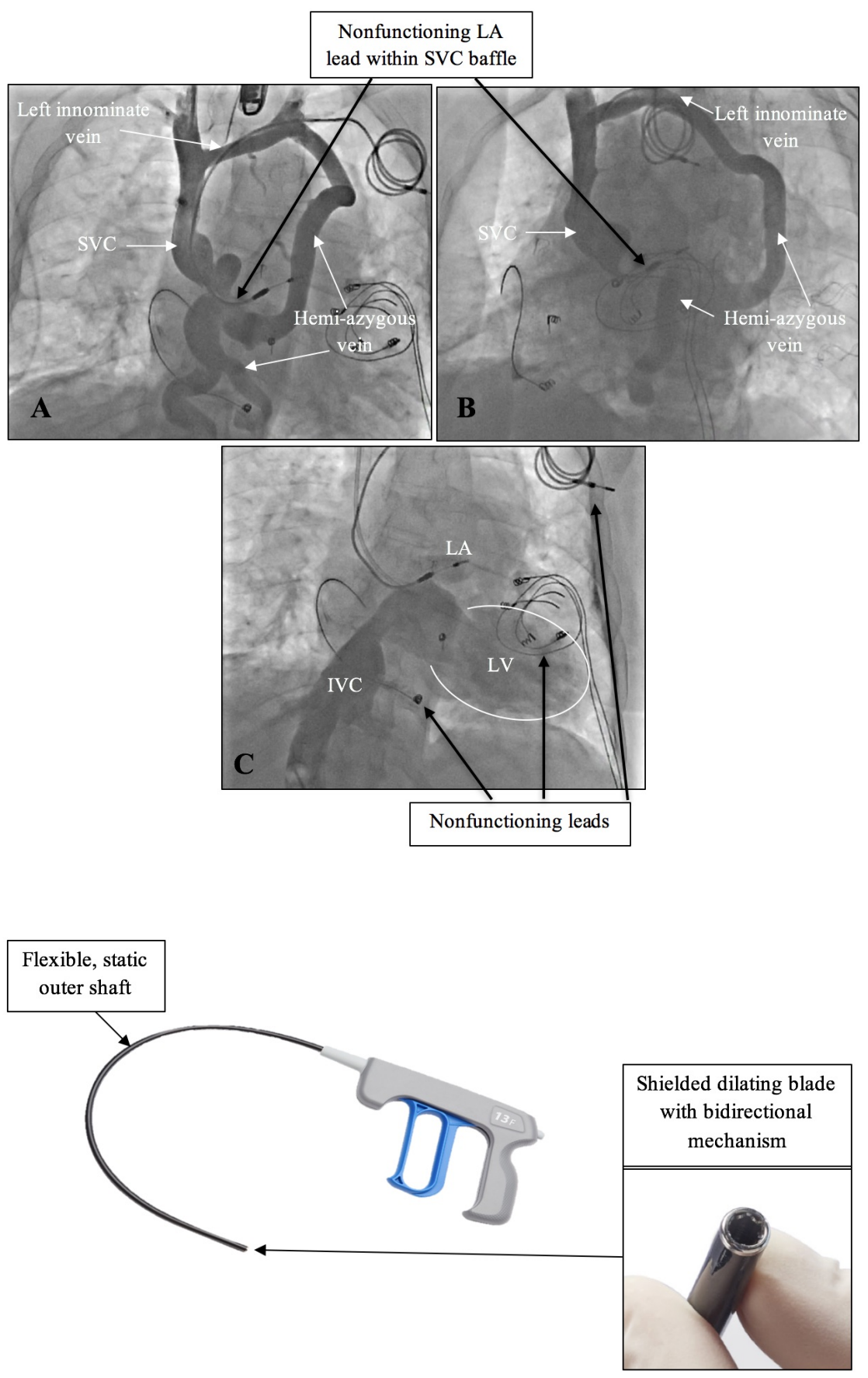


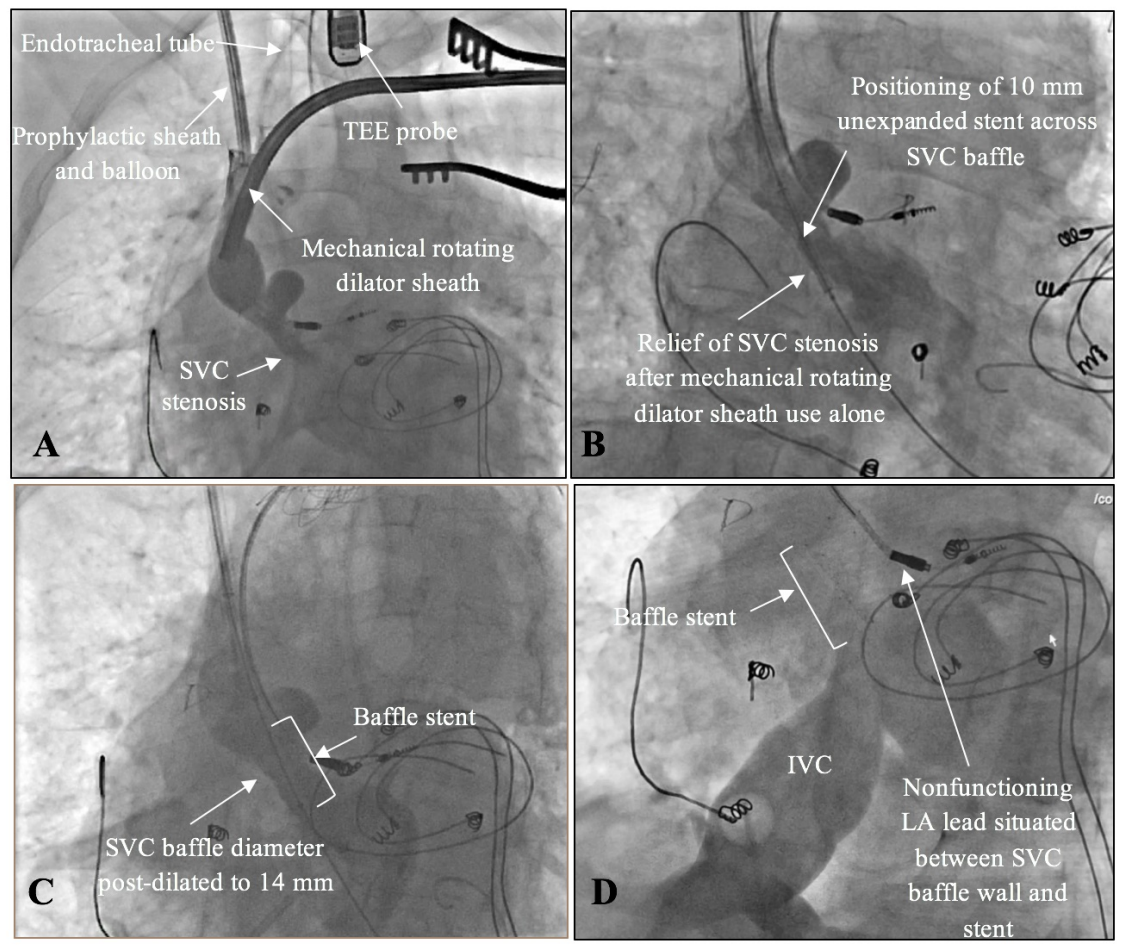

